\title{
On the Comparison of Uncertainty Criteria for Active SLAM
}

\author{
Henry Carrillo, Ian Reid and José A. Castellanos
}

\begin{abstract}
In this paper, we consider the computation of the D-optimality criterion as a metric for the uncertainty of a SLAM system. Properties regarding the use of this uncertainty criterion in the active SLAM context are highlighted, and comparisons against the A-optimality criterion and entropy are presented. This paper shows that contrary to what has been previously reported, the D-optimality criterion is indeed capable of giving fruitful information as a metric for the uncertainty of a robot performing SLAM. Finally, through various experiments with simulated and real robots, we support our claims and show that the use of $D$-opt has desirable effects in various SLAM related tasks such as active mapping and exploration.
\end{abstract}

\section{INTRODUCTION}

A model of the operative environment is an essential requirement for an autonomous mobile robot. The construction of this model requires the solution of at least three basic tasks for a mobile robot, namely localization, mapping and trajectory planning. The intersection of the first two tasks defines a key problem in modern robotics: Simultaneous Localization and Mapping (SLAM).

SLAM is the problem of acquiring on-line and sequentially spatial data of an unknown environment in order to construct a map of it, and at the same time, allows the robot to localize itself in this map.

To integrate the trajectory planning into SLAM allows a mobile robot to perform common tasks such as autonomous environment exploration. This approach is known as active SLAM and specifically refers to the problem of how to give a mobile robot the capability of generating on-line trajectories that simultaneously maximize the accuracy of the map and robot's localization, regarding a SLAM task.

The active SLAM paradigm was first proposed and tested in [1]. Since then, different approaches have been done. e.g. [2] and [3] proposed a discrete and greedy planning methodology. Huang et al. in [4] studied and tested the feasibility of multi-step planning. Continuous states planning but with a discretization in actions space is explored in [5]. Recently, a continuous planning approach in states and actions has been proposed by [6].

To the best of the authors' knowledge, the different approaches that attempt to produce an active SLAM algorithm,

This work has been supported by the MICINN-FEDER project DPI200913710, and research grants BES-2010-033116 and EEBB-2011-44287.

H. Carrillo and J. A. Castellanos are with the Departamento de Informática e Ingeniería de Sistemas, Instituto de Investigación en Ingeniería de Aragón Universidad de Zaragoza, C/ María de Luna 1, 50018, Zaragoza, Spain. \{henry.carrillo, jacaste\}@unizar.es

I. Reid is with the Department of Engineering Science, University of Oxford, Parks Road, Oxford, United Kingdom. ian@robots.ox.ac.uk rely on criteria or metrics that quantify the improvement of the actions taken by the robot (e.g. movements). This improvement is measured relative to $(i)$ the robot and the map localization accuracy, (ii) the area of the map explored or (iii) the time that the robot has been navigating. Specifically, the metrics that relate the improvement of the localization accuracy or the uncertainty related to the movements the robot makes are of high value, because their uses allow the reduction of the map's error, and therefore the probability to accomplish a given task is improved.

Until now the preferred criterion to quantify the localization uncertainty has been the A-optimality criterion $(A-o p t)$. This criterion captures the mean uncertainty of the covariance matrix of a SLAM system. The choice of this criterion in many active SLAM related works such as [7], [8], [5], [9], and [6], among others, had its foundation in the fact that papers such as [10], [11], and [12] reported that (i) the A-opt applied to the problems of planning under uncertainty out performs other well-known criteria such as the D-optimality criterion (D-opt), and (ii) that the $D$-opt for the active SLAM case does not produce a meaningful metric.

However, in the Theory of Optimal Experiment Design (TOED) [13] [14], it is well-known that the use of the $D$ opt has more appealing characteristics than the A-opt or Eoptimality criterion (E-opt). Moreover, Kiefer in [15] demonstrated that the A-opt, D-opt and E-opt are special cases of a general family of uncertainty criteria and therefore they share some properties, but $D$-opt is the only one proportional to the uncertainty ellipse of the estimated parameters, and it is also invariant to re-parametrizations and linear transformations [14].

This paper shows that is indeed possible to obtain a fruitful metric from the D-opt for the particular case of a mobile robot performing SLAM. Additionally, it is shown experimentally, that the use of $D$-opt as a metric for quantifying the uncertainty of the robot and the generated map in an active SLAM context, performs comparably to the A-opt metric popularized by [10], [11] and [12].

The reminder of the paper is structured as follows: section II gives an overview of the active SLAM problem and its connection to the TOED. Section III shows how to compute $D$-opt in order to be compared correctly, and to allow its use in an active SLAM or path planning under uncertainty context. Sections IV and V report several experiments with simulated and real robots that support our claims. Finally, section VI presents the conclusions. 


\section{ACTIVE SLAM}

The SLAM problem does not establish which trajectories a robot has to follow. Usually, they are chosen randomly or beforehand. However, it is well-known that the trajectories selected and the order they are executed by a robot, are critical, among other things, firstly for a rapidly convergence of the uncertainty of a SLAM algorithm, secondly for increasing the area of the environment explored by the robot, and thirdly to improve the possibility of fulfilling tasks.

The integration of the trajectory planning task into SLAM was first proposed in [1] and the term active SLAM referring to the aforementioned integration was coined by [8]. The general idea of active SLAM can be summarized as follows in algorithm 1:

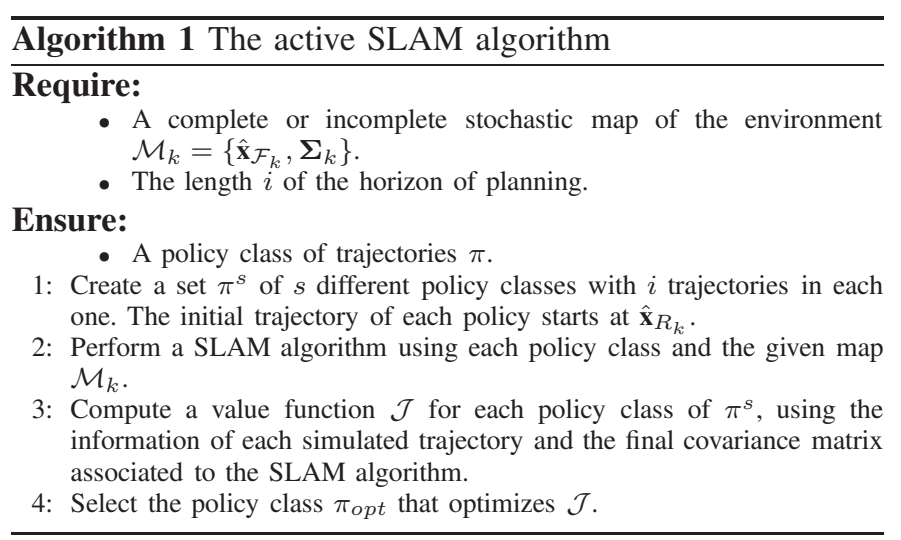

The SLAM approach taken above is based on a probabilistic state-space model, where the robot $R$ and a set of features or landmarks in the environment $\mathcal{F}=\left\{F_{1}, \ldots, F_{n}\right\}$ are represented by a stochastic state vector $\mathbf{x}$ with an estimated mean $\hat{\mathbf{x}}$ and associated covariance matrix $\boldsymbol{\Sigma}$. Furthermore,

$$
\hat{\mathbf{x}}=\left[\begin{array}{c}
\hat{\mathbf{x}}_{R} \\
\hat{\mathbf{x}}_{\mathcal{F}}
\end{array}\right] ; \quad \boldsymbol{\Sigma}=\left[\begin{array}{c|c}
\boldsymbol{\Sigma}_{R R} & \boldsymbol{\Sigma}_{R \mathcal{F}} \\
\hline \boldsymbol{\Sigma}_{\mathcal{F} R} & \boldsymbol{\Sigma}_{\mathcal{F F}}
\end{array}\right]
$$

where $\hat{\mathbf{x}}_{R}$ and $\hat{\mathbf{x}}_{\mathcal{F}}$ are the estimated locations of the robot and the landmarks respectively, $\boldsymbol{\Sigma}_{R R}$ is the covariance matrix of the estimated robot pose (e.g. $x, y, \theta)$ and it has a size of $p \times$ $p$ that is invariant with respect to the time, $\boldsymbol{\Sigma}_{\mathcal{F} \mathcal{F}}$ represents the covariance matrix of the estimated locations of the discovered landmarks and it has a size of $n \times n$ that varies over time. Finally, $\boldsymbol{\Sigma}_{R \mathcal{F}}$ and $\boldsymbol{\Sigma}_{\mathcal{F} R}$ are matrices that encode the crosscovariance of the robot pose and the landmarks estimations. The covariance matrix $\boldsymbol{\Sigma}$ has size $l \times l$, where $l=p+n$, and its value is variable with time. Moreover, it is a positive semi-definitive matrix with eigenvalues $\left\{\lambda_{1}, \ldots, \lambda_{l}\right\}$.

\section{A. The Value Function $\mathcal{J}$}

As mentioned above, the integration of trajectory planning or, what is equivalent, applying the active sensing paradigm [16] [10] to the SLAM problem, involves the optimization of a multi-objective performance criterion or value function $\mathcal{J}$.

This value function is used to decide which trajectories have to be followed by the robot. A definition of this value function can be as follows:

$$
\mathcal{J}=\sum_{i} \alpha_{i} \mathcal{U}_{i}+\sum_{i} \beta_{i} \mathcal{T}_{i}
$$

Where the index $i$ defines the length of the planning horizon (i.e. the numbers of consecutive trajectories planned ahead). The first term, $\mathcal{U}_{i}$ characterizes the expected cost of the uncertainty in the parameters of the system. The second term, $\mathcal{T}_{i}$ includes other expected costs such as trajectory length, navigation time, and energy consumption, among others. Finally, $\alpha$ and $\beta$ are weight coefficients for tuning the parameters and are task dependant.

The $\mathcal{U}_{i}$ term can be further specified as a metric of the associated covariance matrix $\Sigma$ (e.g. the determinant, the trace). This metric needs to encode the robot and the landmarks' estimated locations uncertainty and can be defined as follows:

$$
\mathcal{U}_{i}: \Sigma \rightarrow \mathbb{R}
$$

The different ways to compute the above metric and their properties in relation to the goals of the active SLAM approach is the target of the following sections of this paper. Moreover, a clarification in the computation of one of them is pointed out in section III.

The second term $\mathcal{T}_{i}$, as done previously, can be further specified and constrained as a metric that represents the cost of performing a free collision trajectory $\Gamma$ by the robot,

$$
\mathcal{T}_{i}: \Gamma \rightarrow \mathbb{R}
$$

This metric can be constrained to be a function only of the distance travelled, since its cost is directly related to the power and navigation time of the robot while it performs a task.

Finally, summarizing all the above definitions, the statement of the active SLAM problem can be formulated as: the task of choosing a single or multiple step policy class $\pi$ of robot's trajectories that optimize a value function $\mathcal{J}$.

\section{B. Theory of Optimal Experiment Design and Active SLAM}

In the Theory of Optimal Experiment Design (TOED) [13] [14], a single trial of an experiment is the process of changing the input parameters of a system perturbed with unknown noise, with the purpose of observing the variation in the output parameters. In this context, the particular values of the input parameters are known as a particular design $\xi$.

In the active SLAM context, the $\xi$ design is a particular policy class $\pi$ commanded to the robot, the unknown noise is the commonly assumed zero mean Gaussian noise and the variation of the parameters is encoded in the covariance matrix $\Sigma$.

Based on the TOED, it is possible to know if a design $\xi_{1}$ is better than a design $\xi_{2}$ [13] [14]. Applying this concept in the active SLAM context, a policy class $\pi_{1}$ is better in terms of uncertainty than a policy class $\pi_{2}$ if :

$$
\operatorname{Cov}\left(\pi_{1}\right)-\operatorname{Cov}\left(\pi_{2}\right) \in \operatorname{NND}(l)
$$


Where $\operatorname{Cov}\left(\pi_{i}\right)$ is the covariance matrix of size $l \times l$ after the robot has followed $\pi_{i}$ and $\operatorname{NND}(l)$ stands for the group of non-negative definite matrices of size $l \times l$. NND matrices are also known as positive semi-definite matrices [14].

As this criterion only tells if a policy class is better than another but does not quantify how much, it is advantageous to define a function $\phi$ that maps a NND covariance matrix of size $l \times l$ to a scalar,

$$
\phi: \mathrm{NND}(l) \rightarrow \mathbb{R}
$$

This function has to capture the idea of whether or not the uncertainty of a covariance matrix is large or small. Moreover, this function has to be positive homogeneous, isotonic (i.e. order preserving) and concave [14].

A compendium of functions fulfilling the above requirements can be found in [13] or [14]. Among the most commonly used functions or uncertainty criteria are the Aoptimality criterion $(A-o p t)$, the D-optimality criterion (D-opt) and the E-optimality (E-opt) criterion.

\section{UNCERTAINTY CRITERIA FOR ACTIVE SLAM}

In the planning under uncertainty or active SLAM context, previous works such as [10], [11] and [12], have done comparisons between uncertainty criteria, in order to determine if there is a criterion that for that specific task, converges faster to a desired solution. In all the aforementioned papers, the $D$-opt - defined by them as the determinant of the covariance matrix - has been disregarded as a fruitful metric for mainly two reasons:

i) The D-optimality criterion does not allow the checking of task completion as the A-optimality criterion does.

ii) The D-optimality criterion can be driven rapidly to zero, so no fruitful information is provided by this criterion.

The authors believe that the above two reasons are misconceptions stemming from a misuse of the TOED.

For the first reason, the misuse lies in that the determinant of a matrix $l \times l$ is homogeneous of degree $l$; hence the comparison of the determinant of a matrix $l \times l$ and a matrix $m \times m$ is unfair. Specifically in the case of a SLAM system this is relevant, because the size of the covariance matrix varies over time, so the evolution of an uncertainty criterion based on determinants has to be normalized in order to be compared fairly [14].

Recently, Vidal-Calleja et al [17] intuited this, and proposed a solution that needs to suppose the maximum number of landmarks in the environment and initialize its covariance with a constant number. This solution is effective to fairly compare the determinant as the matrix size does not vary in time, but adds complexity to the computation of the metric and fails if the number of landmarks is greater than the initial assumption.

A proper solution, as pointed out by [14], is to take the $l^{t h}$ root of the determinant of $\boldsymbol{\Sigma}$ (with size $l \times l$ ) before making any comparison. This solution rises naturally if the D-opt is derived from the family of uncertainty criteria proposed by Kiefer in [15],

$$
\phi_{p}(\xi)=\left[l^{-1} \operatorname{trace}\left(\boldsymbol{\Sigma}^{p}(\xi)\right)\right]^{1 / p}
$$

This family of uncertainty criteria is valid in the range of $0<p<\infty$ for a covariance matrix $(\boldsymbol{\Sigma})$ of size $l \times l$ associated to a design $\xi(e . g . \pi)$. Moreover, the case $\phi_{1}$ and the boundary cases $\phi_{0}$ and $\phi_{\infty}$ are the already known A, D and E-optimality criteria, respectively.

Taking the above into account, the normalized D-optimality criterion proposed by Kiefer is,

$$
\begin{aligned}
\phi_{0}(\pi)=\lim _{p \rightarrow 0+} \phi_{p}(\pi) & =[\operatorname{det}(\boldsymbol{\Sigma}(\pi))]^{1 / l} \\
& =\left(\prod_{k=1, \ldots, l} \lambda_{k}\right)^{1 / l}
\end{aligned}
$$

The misuse of TOED for the second reason, usually used to disregard the $D$-opt, lies in the fact that this criterion considers the global variance. Geometrically, this means the volume of a n-dimensional ellipsoid [13]. The latter implies that estimated parameters with low uncertainty will produce very low value of $D$-opt, hence making its computation prone to round-off errors.

Specifically in the SLAM case, as the landmarks get correlated the eigenvalues of $\boldsymbol{\Sigma}$ become quite small values near to zero. A zero eigenvalue would mean that without doubt the position of a landmark is known, but this does not happen in practice. Examples of the above are presented in section IV, where we reported several experiments with simulated and real robots. Regarding the computation of the determinant, it is possible that a small value of an eigenvalue can cause a round-off error in the computation, so the D-opt gets stuck at zero. One way to overcome this issue is to use the logarithmic space to compute the determinant, using a similar approach as in [13]. Thus, the resulting equation to compute the criterion would be,

$$
\exp \left(\log \left([\operatorname{det}(\boldsymbol{\Sigma}(\pi))]^{1 / l}\right)\right)
$$

Summarizing, for the particular case of measuring the uncertainty of a SLAM system, the D-opt should be computed using the definition of Kiefer [15] and as presented in (9).

In the following, two experiments are presented in order to (i) support the claims about the computation of the Doptimality criterion of a SLAM system, and, (ii) to point out some properties of the D-optimality criterion. The first experiment investigates the evolution of different uncertainty metrics in simulated and real robots performing SLAM. The second experiment is related to performing active SLAM using solely the uncertainty as a guiding factor.

\section{FIRST EXPERIMENT: ON THE COMPUTATION}

With the aim of showing that it is feasible to compute the D-opt in a robot performing SLAM, in the following, the evolution of the aforementioned uncertainty criterion is computed for simulated and real robots performing SLAM. For completeness, the A-opt, E-opt, the determinant of the 


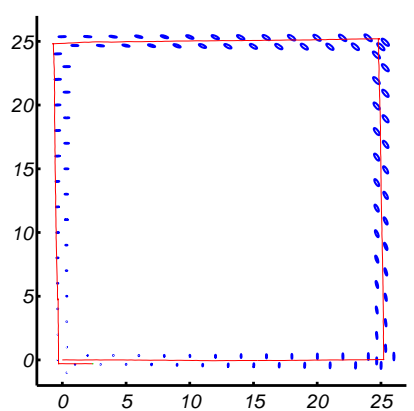

Fig. 1. Resulting stochastic map $(25 \times 25 \mathrm{~m})$ for the experiment with a simulated robot in an indoor environment. In red is the estimated trajectory of the robot and in blue is the graphical representation of the covariance for each landmark.

covariance matrix, entropy and mutual information are also computed.

In each of the following experiments the aforementioned uncertainty criteria are computed at each step update of the covariance matrix $\boldsymbol{\Sigma}$ associated to $\hat{\mathbf{x}}_{R}$ and $\hat{\mathbf{x}}_{\mathcal{F}}$.

\section{A. Simulated Robot in an Indoor Environment}

The simulation environment was created using $\mathrm{C}++$ and the Mobile Robot Programming Toolkit (MRPT) v0.9.4. The data of the covariance matrix were gathered while the robot was performing EKF-SLAM with a predefined trajectory, within a map with static landmarks and using a limited range sensor.

Specifically, the robot was moving at $0.3 \mathrm{~m}$ per step and travelled along a square-shaped trajectory of $25 \times 25 \mathrm{~m}$. The navigation environment was composed of 2-D point features, located in both sides of the trajectory with a distribution of 1.8 feature $/ \mathrm{m}$. The robot was equipped with a range-bearing sensor with a frontal field of view of $360^{\circ}$ and a maximum range of $3 \mathrm{~m}$. Synthetic errors, with a Gaussian distribution, were generated for the odometry model of the robot (standard deviations of $0.1^{\circ}$ in orientation and $0.2 \mathrm{~m}$ per $\mathrm{m}$ in displacement) and the sensor measurements (standard deviations of $0.125^{\circ}$ in orientation and $1 \mathrm{~cm}$ per $\mathrm{m}$ in range), but known data association is assumed. The resulting stochastic map after one loop is shown in Fig. 1.

Fig. 2 shows the evolution of the different criteria as stated above. Each point of the evolution gives an indication of the amount of uncertainty the SLAM system has at that step. As expected, once the robot starts navigating, the uncertainty related to the landmarks and robot's localization starts increasing. The evolution of the tested criteria behaves similarly at this stage.

Around the step 350 a loop closing event occurred, and therefore a decrease in the uncertainty of the system is produced as expected. This drop is sensed by all the metrics but at different magnitudes, A-opt and E-opt had a major reduction, but $D$-opt had a minor one.

The difference in magnitude is due to the opposite definition of the metrics. D-opt in general, takes into account the uncertainty of each element of the system multiplicatively, i.e. every element has an equal chance to contribute to the uncertainty. This definition allows encompassing the global uncertainty in the D-optimality criterion.

On the other hand, A-opt gives independent and additive contribution to each element of uncertainty. Giving the possibility of a single component of the system to drive the whole uncertainty. In fact, in Fig. 2a and Fig. 2b, A-opt and E-opt resemble in shape and scale, thus giving a numerical example, although qualitative, of the above, as E-opt represents the value of the single maximum eigenvalue. Moreover, the correlation between $A$-opt and $E$-opt is 0.9655 , giving a quantity value of its resemblance.

Fig. 2d shows an example of computing the determinant of the covariance matrix as reported in [10], [11] or [12], as can be seen after few steps -in this case 8- the value of the criterion goes to zero. In contrast, Fig $2 c$ shows an example of meaningful values of uncertainty using the logarithmic based computation method presented in (9).

\section{B. Real Robot in an Indoor Environment: DLR Dataset}

In this experiment the Deutsches Zentrum fur Luft und Raumfahrt (DLR) dataset [18] is used. This dataset was recorded at the DLR with a mobile platform. The environment (see Fig. 3) is a typical office indoor environment and covers a region of $60 \times 45 \mathrm{~m}$. To estimate the trajectory and the map of the environment an EKF based SLAM algorithm coded in python is used.

Fig. 4 shows the evolution of the different uncertainty criteria associated to the uncertainty of the robot and landmarks for the DLR dataset that has a path length of approximately 505 meters.

\section{Discussion}

The above results give numerical examples about the feasibility of computing the D-opt in the SLAM context in simulated and real data. Also, the results give some insights between the relation among the A-opt and E-opt. Although this relation (shape and magnitude of the plots) is qualitative, a quantitative relation via the correlation of the data can be obtained.

The correlation between the A-opt and the E-opt for all the experiments has a mean of $0.9798 \pm 4.0612 \times 10^{-4}$. The latter means that exist a strong relation between these two criteria in the SLAM context. Moreover, based on the definition of the $E$-opt, the uncertainty measured by the A-opt is dominated by a single eigenvalue. In our context, the above implies that a single feature - in the case of a probabilistic feature based SLAM - can drive the complete SLAM uncertainty. The effect of the above property could lead an active SLAM algorithm using an A-opt based metric to get stuck in a local minima. An example of this is shown in the next experiment.

For the above experiments, the A-opt and D-opt correlation has a mean of $0.6454 \pm 0.1359$, which means that exist a correlation but neither is weak or strong. Moreover, it gives an example of the main characteristic of the criteria according to the TOED: The A-opt measures the mean of the 


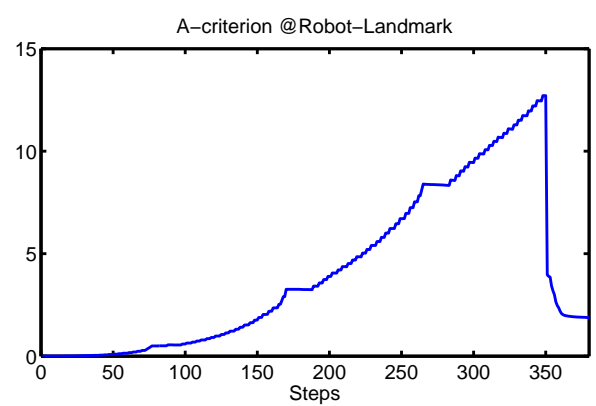

(a)

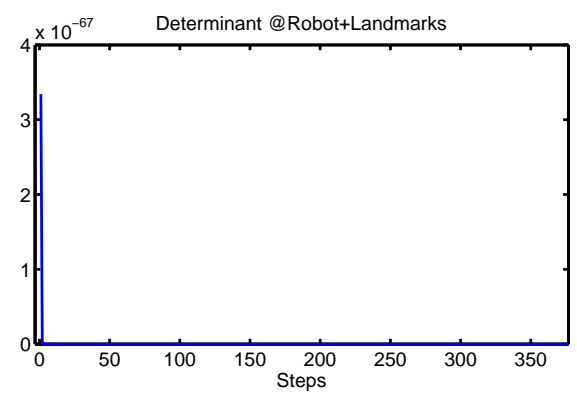

(d)

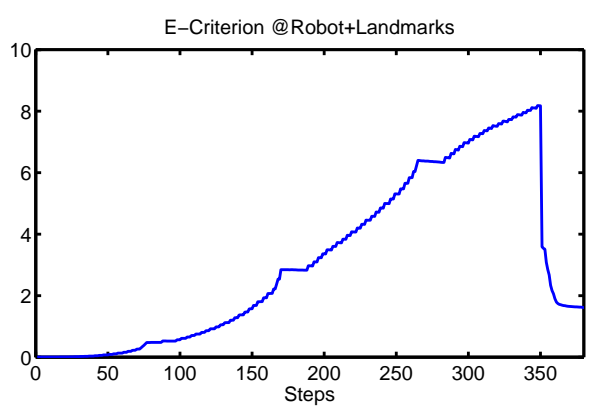

(b)

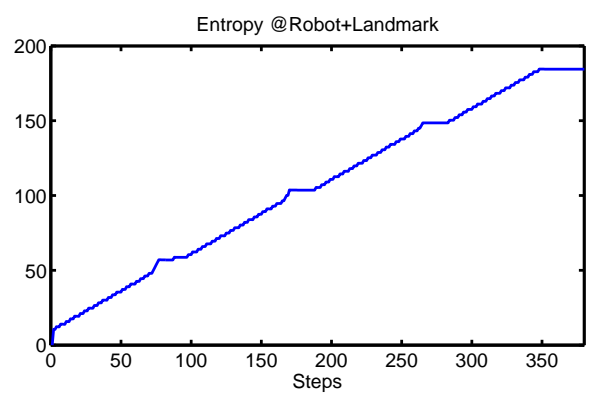

(e)

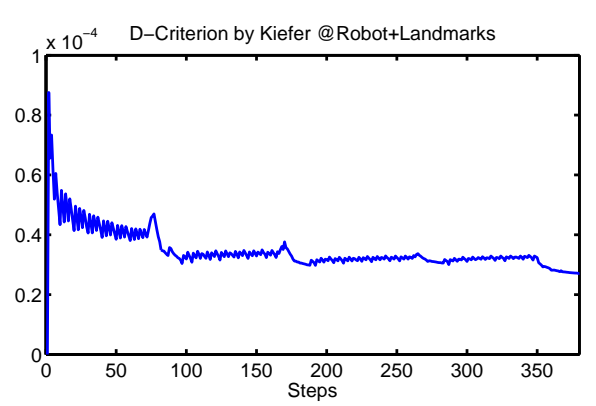

(c)

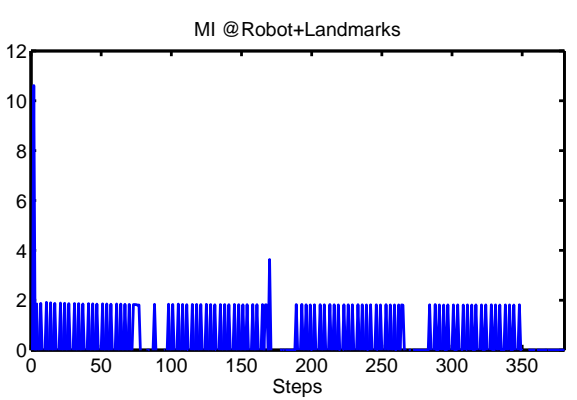

(f)

Fig. 2. (a)-(f) Evolution of the A-opt, E-opt, D-opt, determinant, entropy and MI for the experiment with a simulated robot in an indoor environment

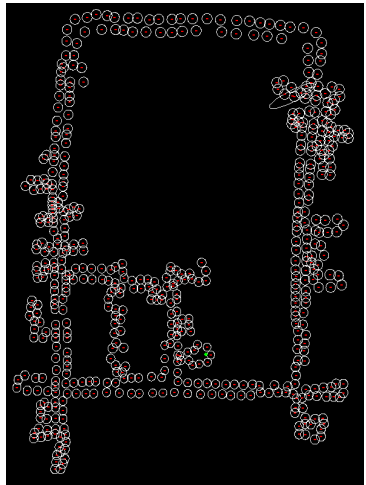

(a)

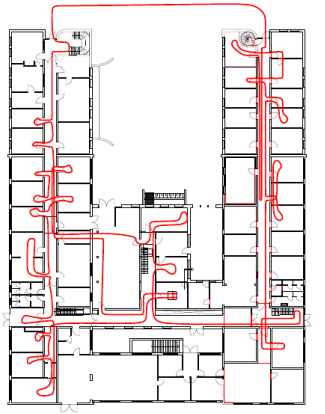

(b)

Fig. 3. (a) Resulting stochastic map with uncertainty regions for each landmark. (b) Blueprint of the environment with a superimposed sketch of the trajectory

uncertainty and the $D$-opt measures the complete dimension of the uncertainty (e.g. Area in a 2D case).

\section{SECOND EXPERIMENT: ACTIVE APPROACH}

In this experiment we perform a comparison between an active SLAM approach driven by the $A$-opt, D-opt and entropy. The active SLAM approach used follows the algorithm outlined in section II, therefore assumes a priori and probably incomplete, stochastic map of the environment. This map is generated by commanding the robot to follow a predefined trajectory in the environment, while performing EKF-SLAM. Once the predefined trajectory is completed, the robot begins the performance of active SLAM and therefore starts planning autonomously trajectories that achieve an accurate map.

Each time the robot is planning which trajectory it has to follow, in order to fulfil the active SLAM objectives, it needs to consider every possible path in the navigation environment. In order to make the problem computationally tractable, the possible destinations are constrained to positions near the landmarks already discovered.

If each time we plan only the next movement, this is known as greedy search or one step look-ahead [4]. It is also possible to plan several steps ahead that will result, as has been pointed out by [4], in a faster convergence of the active SLAM goals but with an increase in the complexity of the computation. Independent of the one step look-ahead or multi-step lookahead planning, each time the next movement is chosen as the one that minimizes an uncertainty metric, in this case the value of $A$-opt or D-opt or entropy related to the SLAM.

In this experiment, the paths follow autonomously for the robot are generated via an $A^{*}$ based path planner. Specifically the environment is discretized and the only forbidden areas are the positions of the landmarks. Two test environments were used for this experiment: the first test environment consists of a 30x30 meters obstacle free square area with 104 landmarks distributed around the perimeter of a 25 meters square. The second test environment consists of a 20x20 meters obstacle free square area with 72 landmarks distributed on the perimeter of a 15 meters square. The Mean Squared Error (MSE) between the two initial stochastic maps has a ratio of 9.65 , with the first environment having a bigger MSE. The initial position of the robot is $(\mathrm{X}=1, \mathrm{Y}=0)$ in both environments. The ground truth position of the landmarks and their estimated positions from the EKF-SLAM are depicted in Fig. 5. 


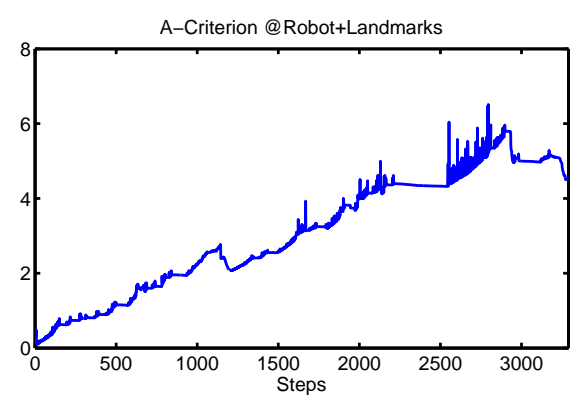

(a)

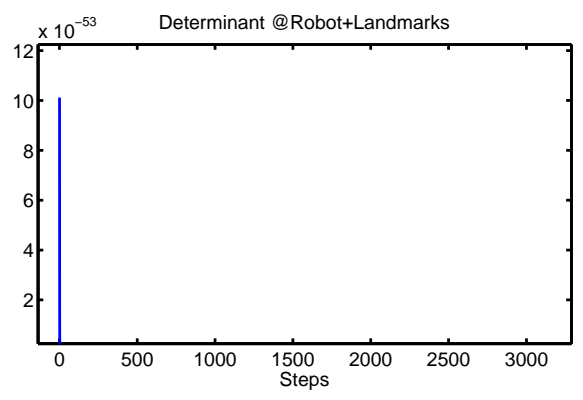

(d)

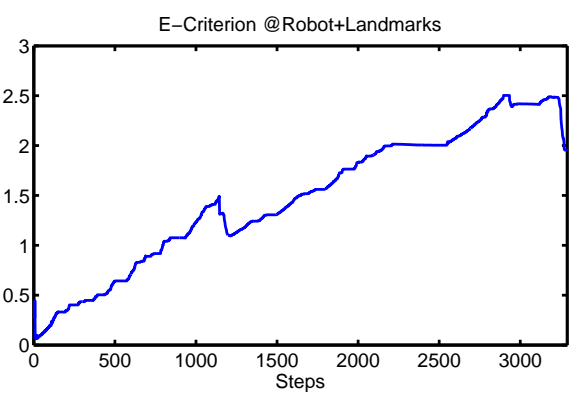

(b)

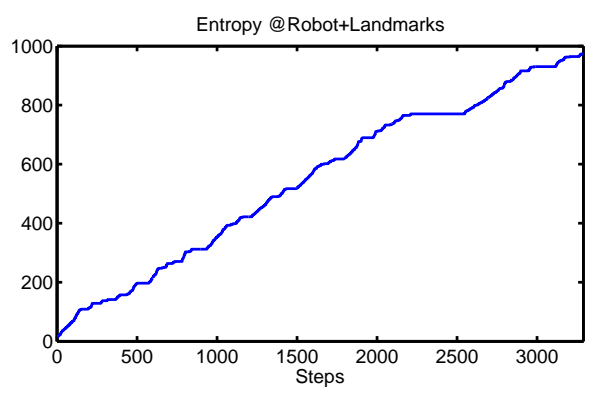

(e)

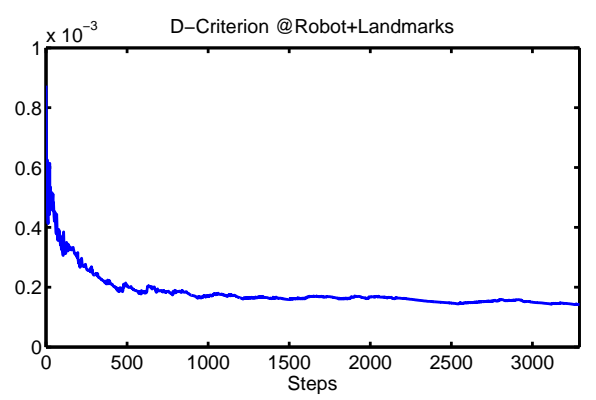

(c)

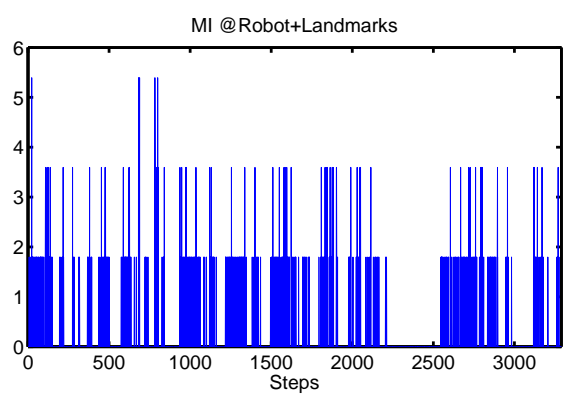

(f)

Fig. 4. (a)-(f) Evolution of the A-opt, E-opt, D-opt, determinant, entropy and MI for the experiment using the DLR dataset

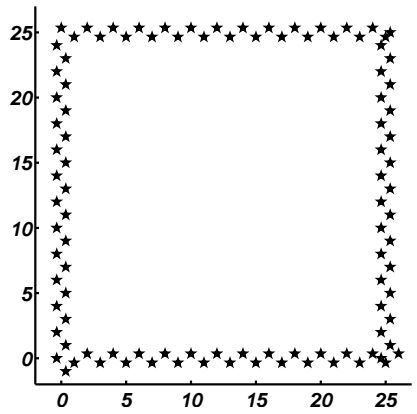

(a)

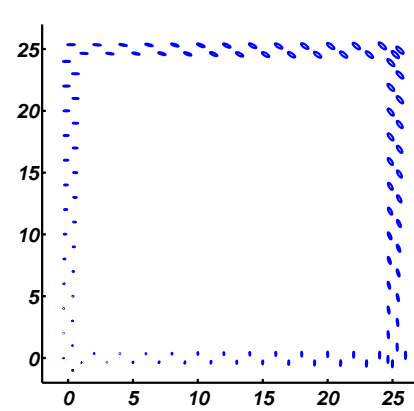

(b)
Fig. 5. (a) Ground truth of the landmarks and (b) initial stochastic map of the $30 \times 30 \mathrm{~m}$ test environment

The strategy for active SLAM described above can be summarized in the following steps:

- Hallucinate paths from the current estimated position of the robot to all the landmarks, except those which are below a radius of $X$ (i.e. 1) meters from the current estimated position.

- Measure the uncertainty at the end of each hallucinated path.

- Select the path that produced the lowest uncertainty according to the chosen metric.

- If the number of path planned is greater than $i$ (i.e. 100), exit. In any other case, execute again.

\section{A. One Step Look-Ahead Results}

Performing active SLAM with a one-step look-ahead approach leads to completely different trajectories using the $A$ - opt and D-opt. The A-opt plans trajectories with a distinctive local behaviour, while the $D$-opt plans trajectories more globally, often revisiting previous landmarks. Regarding the entropy, this generates paths similar to the D-opt.

An example of the above behaviour is illustrated in Fig. 6. There, the active SLAM starts after the robot has executed one loop (i.e. $\mathrm{X}=1, \mathrm{Y}=0$ ) and has an estimation of all the landmarks in the environment. The resulting trajectories for the $A$-opt, $D$ $o p t$ and the entropy are shown in Fig. 6a, Fig. 6b and Fig. 6c, respectively. Each generated trajectory is identified by a different colour. A video of the incremental construction of each trajectory can be seen in http://webdiis.unizar.es/ hcarri/1.avi.

In addition to the above qualitative assessment of the effect derived by using each criterion, we can quantify the effect of using each criterion by measuring the quality of its resulting maps.

To measure the quality of the map we use the guidelines proposed in [19] that urge for the use of the MSE and $\chi^{2}$ together in the assessment of the maps quality generated by a SLAM algorithm.

In order to compare the three criteria, we compute the ratio between them for each quality metric at each update step of the active algorithm. Therefore we have the $A$-opt/D-opt ratio, the A-opt/entropy ratio and the entropy/D-opt for the MSE and $\chi^{2}$ metric.

Fig. 7 presents the result of 10 Monte Carlo runs for each ratio related to the MSE and $\chi^{2}$ metric of the $30 \times 30$ $\mathrm{m}$ test environment. Respectively, Fig. 8 presents the same information for the 20x20 m environment.

Finally, Fig. 9 shows the resulting path for the active SLAM strategy presented in this section using a limit of 10000 steps 


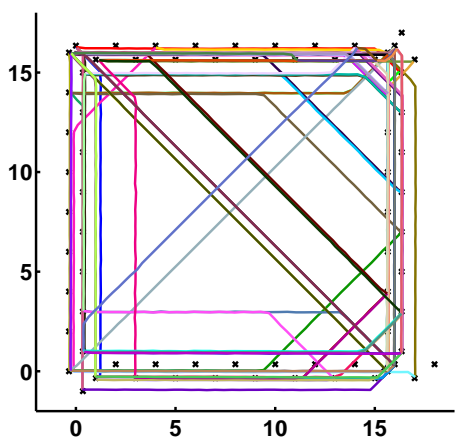

(a)

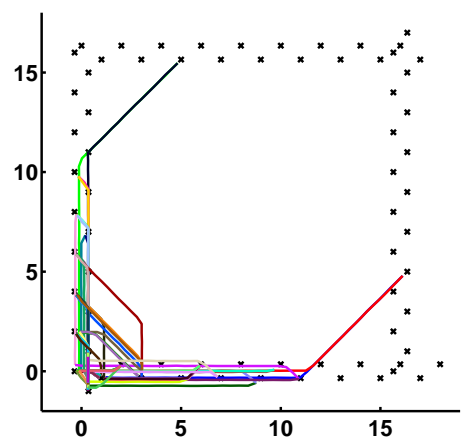

(b)

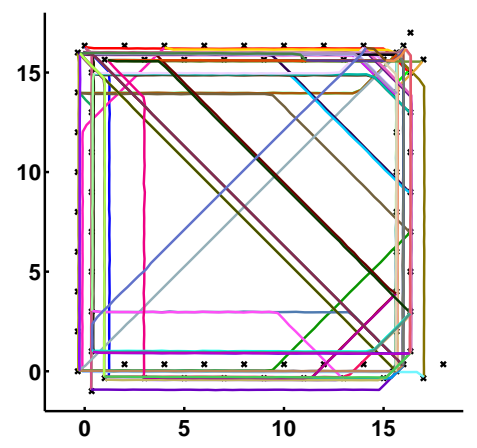

(c)

Fig. 6. Resulting paths from each uncertainty metric: (a) D-opt, (b) A-opt and (c) Entropy. Each colour represents an executed path. The planning area was $20 \times 20 \mathrm{~m}$.

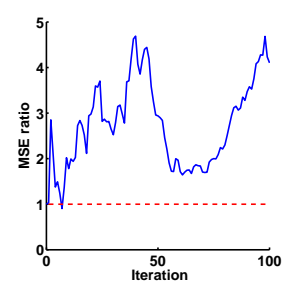

(a) A-opt/D-opt

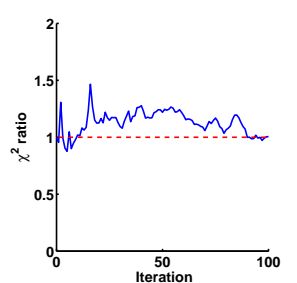

(d) A-opt/D-opt

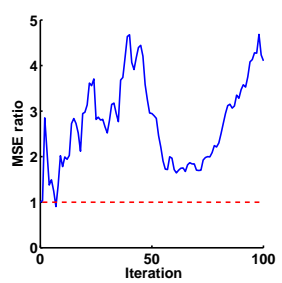

(b) A-opt/Entropy

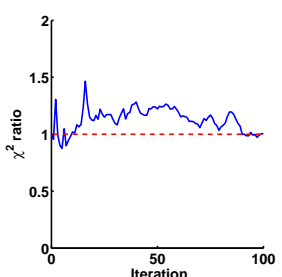

(e) A-opt/Entropy

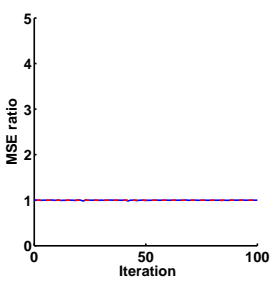

(c) Entropy/D-opt

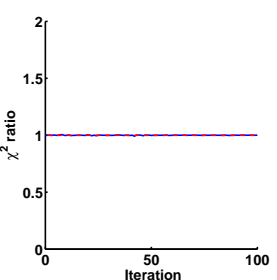

(f) Entropy/D-opt

Fig. 7. Evolution of the MSE ((a)-(c)) and $\chi^{2}$ ((d)-(f)) ratios related to the map $(30 \times 30 \mathrm{~m})$ after each active step. The ratios are computed for each possible uncertainty metric combination. The average of 10 Monte Carlo runs is depicted for each ratio.

and a continuous path planner based on an attractor/repulsion technique. This last experiment illustrates another example of the quasi-opposite behaviour of an active SLAM strategy using the A-opt and D-opt.

\section{B. Discussion}

An explanation of the difference in the path planning behaviours due to the $A$-opt or $D$-opt used relies on the definition of the metric itself. As pointed out in the previous section, $D$-opt encompasses the global uncertainty therefore revisiting previous landmarks (closing the loop) helps in decreasing the value of the metric. On the other hand, A-opt criterion can be driven by a single eigenvalue, and therefore the uncertainty of the covariance matrix can get stuck in a local minimum.

Regarding the quality of the maps, the results show an advantage in the use of $D$-opt and entropy over the A-opt. Also in this specific experiment the $D$-opt and entropy share similar results. This similarity does not come as a surprise, because

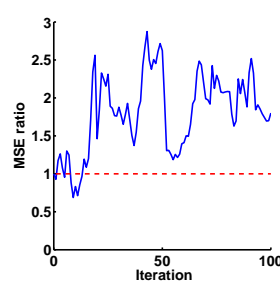

(a) A-opt/D-opt

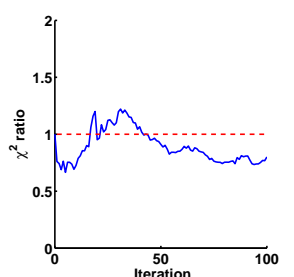

(d) A-opt/D-opt

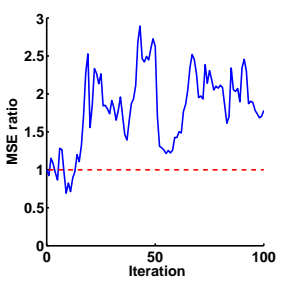

(b) A-opt/Entropy

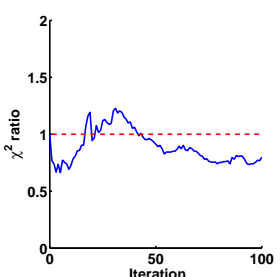

(e) A-opt/Entropy

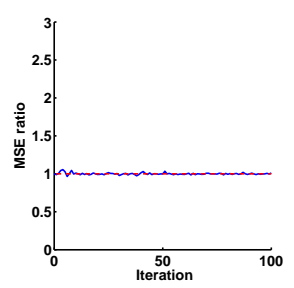

(c) Entropy/D-opt

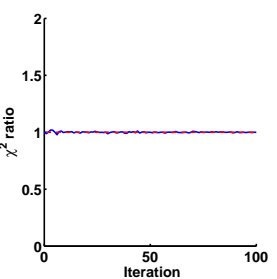

(f) Entropy/D-opt
Fig. 8. Evolution of the MSE ((a)-(c)) and $\chi^{2}((\mathrm{~d})-(\mathrm{f}))$ ratios related to the map $(20 \times 20 \mathrm{~m})$ after each active step. The ratios are computed for each possible uncertainty metric combination. The average of 10 Monte Carlo runs is depicted for each ratio.

the EKF-SLAM assumed gaussianity as well the noise used in the experiment, therefore the D-opt and the entropy have an explicit relationship through the determinant as can be seen comparing (9) and the entropy of a multidimensional Gaussian distribution $\left(\right.$ i.e. $\left.\mathcal{N}_{n}(\boldsymbol{\mu}, \boldsymbol{\Sigma})\right)$ :

$$
H(x)=\frac{1}{2} \log (2 \pi e)^{n}|\boldsymbol{\Sigma}|
$$

\section{CONCLUSION}

In this paper a clarification on the use and computation of the D-optimality criterion for a covariance matrix with variable size in time, in order to make comparisons of uncertainty evolution in a SLAM context, is presented. This paper highlights that computing the D-optimality criterion in the SLAM context as reported in previous works such as [10], [11] and [12] leads to wrong results because it does not take into account the change in dimensionality of the determinant. Instead of 


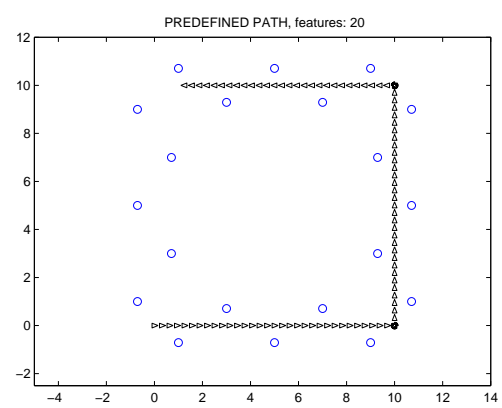

(a)

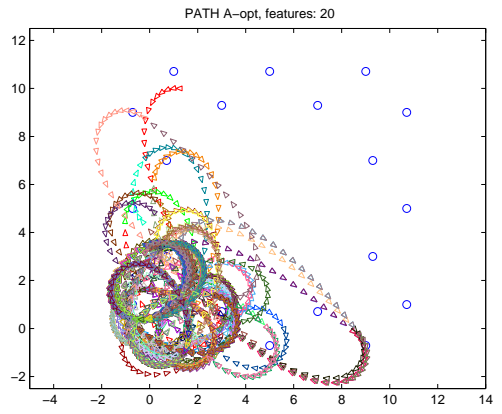

(b)

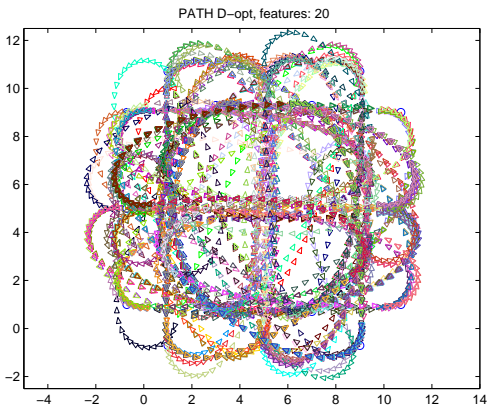

(c)

Fig. 9. Resulting trajectories for a 10000 steps active SLAM simulation. (a). Predefined trajectory and landmarks ground truth. (b). A-opt based active SLAM. (c). D-opt based active SLAM. This figure is best viewed in colour.

the above definition, a method that produces fruitful results is the one proposed by Kiefer [15]. Furthermore, a solution for the problem of round-off errors in the computation of the Doptimality criterion is achieved by proposing its computation in the logarithmic space.

This paper demonstrates via several experiments with simulated and real robots the above claims, and point out appealing characteristics (e.g. encompassing global uncertainty) for the use of D-optimality criterion as a measurement of the uncertainty of a SLAM system. Besides, it is shown that the use of D-optimality criterion, instead of the A-optimality criterion, to drive an active SLAM approach seems more rewarding towards the fulfilling of the active SLAM objectives. Also in the active SLAM context is shown through examples the similarity of guiding a greedy active SLAM strategy with the $D$-opt and the entropy.

Finally, with the clarification reported in this paper, the $D$ opt rises as an alternative to quantify the uncertainty of a SLAM algorithm. Its use has a strong background from the TOED and its properties allow it to be used instead of the commonly used A-opt.

As a future work, firstly we aim at developing a more complex guiding factor for the active SLAM strategy that will include beside the uncertainty, time and obstacle constraints. Secondly, we want to include within the assumptions of the active SLAM, dynamic landmarks and obstacles.

\section{REFERENCES}

[1] H. J. S. Feder, J. J. Leonard, and C. M. Smith, "Adaptive Mobile Robot Navigation and Mapping," The International Journal of Robotics Research, vol. 18, no. 7, pp. 650-668, 1999.

[2] A. Makarenko, S. Williams, F. Bourgault, and H. Durrant-Whyte, "An experiment in integrated exploration," in IEEE / RSJ Int. Conf. on Intelligent Robots and Systems, vol. 1, 2002, pp. 534-539.

[3] C. Stachniss, G. Grisetti, and W. Burgard, "Information Gain-based Exploration Using Rao-Blackwellized Particle Filters," in Proceedings of Robotics: Science and Systems, Cambridge, USA, June 2005.

[4] S. Huang, N. Kwok, G. Dissanayake, Q. Ha, and G. Fang, "Multi-Step Look-Ahead Trajectory Planning in SLAM: Possibility and Necessity," in IEEE Int. Conf. on Robotics and Automation, Apr 2005, pp. 1091 1096.

[5] T. Kollar and N. Roy, "Trajectory Optimization using Reinforcement Learning for Map Exploration," The International Journal of Robotics Research, vol. 27, no. 2, pp. 175-196, 2008.
[6] R. Martinez-Cantin, N. de Freitas, E. Brochu, J. Castellanos, and A. Doucet, "A Bayesian exploration-exploitation approach for optimal online sensing and planning with a visually guided mobile robot," Autonomous Robots, vol. 27, pp. 93-103, 2009.

[7] T. Kollar and N. Roy, "Using reinforcement learning to improve exploration trajectories for error minimization," in IEEE Int. Conf. on Robotics and Automation, May 2006, pp. 3338 -3343.

[8] C. Leung, S. Huang, and G. Dissanayake, "Active SLAM using Model Predictive Control and Attractor based Exploration," in IEEE / RSJ Int. Conf. on Intelligent Robots and Systems, Oct 2006, pp. 5026 -5031.

[9] D. Meger, I. Rekleitis, and G. Dudek, "Heuristic search planning to reduce exploration uncertainty," in IEEE / RSJ Int. Conf. on Intelligent Robots and Systems, Sep 2008, pp. $3392-3399$.

[10] L. Mihaylova, T. Lefebvre, H. Bruyninckx, K. Gadeyne, and J. De Schutter, "A Comparison of Decision Making Criteria and Optimization Methods for Active Robotic Sensing" in Numerical Methods and Applications, ser. Lecture Notes in Computer Science. Springer Berlin / Heidelberg, 2003, vol. 2542, pp. 316-324.

[11] R. Sim and N. Roy, "Global A-Optimal Robot Exploration in SLAM," in IEEE Int. Conf. on Robotics and Automation, Apr 2005, pp. 661 666.

[12] T. Lefebvre, H. Bruyninckx, and J. De Schutter, "Task Planning With Active Sensing For Autonomous Compliant Motion," The International Journal of Robotics Research, vol. 24, no. 1, pp. 61-81, 2005.

[13] A. Pázman, Foundations of Optimum Experimental Design (Mathematics and its Applications). Springer, 1986.

[14] F. Pukelsheim, Optimal Design of Experiments (Classics in Applied Mathematics). Society for Industrial and Applied Mathematics, 2006.

[15] J. Kiefer, "General Equivalence Theory for Optimum Designs (Approximate Theory)," The Annals of Statistics, vol. 2, no. 5, pp. pp. 849-879, 1974.

[16] R. Bajcsy, “Active perception,” Proceedings of the IEEE, vol. 76, no. 8, pp. 966-1005, 1988

[17] T. Vidal-Calleja, A. Sanfeliu, and J. Andrade-Cetto, "Action Selection for Single-Camera SLAM," Systems, Man, and Cybernetics, Part B: Cybernetics, IEEE Transactions on, vol. 40, no. 6, pp. 1567 -1581, Dec 2010.

[18] J. Kurlbaum and U. Frese, "DLR Spatial Cognition Data Set," December 2008. [Online]. Avalible: http://www.informatik.uni-bremen.de/agebv/en/DlrSpatialCognitionDataSet

[19] E. Olson and M. Kaess, "Evaluating the performance of map optimization algorithms," in Robotics: Science and Systems, Workshop on Good Experimental Methodology in Robotics, June 2009. 